\title{
Study of Filtration in Polyethylene Fibers used in Masks for Protection Against SARS-CoV-2 via Luminescent Aerosolized Silicon Nanoparticles
}

\section{Ayman Rezk}

Khalifa University of Science and Technology

Juveiriah Ashraf

Khalifa University of Science and Technology

Wafa Alnaqbi

Khalifa University of Science and Technology

Sabina Abdul Hadi

University of Dubai

Ghada Dushaq

New York University Abu Dhabi

Aisha Alhammadi

Khalifa University of Science and Technology

Tala El Kukhun

University of Toronto

Mahmood Rasras

New York University Abu Dhabi

Ahmad Nusair

Cleveland Clinic

Munir Nayfeh

University of Illinois at Urbana Champaign

Ammar Nayfeh ( $\square$ ammar.nayfeh@ku.ac.ae)

Khalifa University of Science and Technology

\section{Research Article}

Keywords: Commercial polyethylene (PE), coronavirus , SARS-CoV-2, nanoparticles

Posted Date: April 6th, 2021

DOI: https://doi.org/10.21203/rs.3.rs-366914/v1 
License: (c) (i) This work is licensed under a Creative Commons Attribution 4.0 International License. Read Full License 


\title{
Study of Filtration in Polyethylene Fibers used in Masks for Protection Against SARS-CoV-2 via Luminescent Aerosolized Silicon Nanoparticles
}

\author{
Ayman Rezk', Juveiriah M. Ashraf', Wafa Alnaqbi' ${ }^{1}$, Sabina Abdul Hadi², \\ Ghada Dushaq $^{3}$, Aisha Alhammadi', Tala El Kukhun ${ }^{1,4}$, Mahmood Rasras ${ }^{3}$, \\ Ahmad R. Nusair ${ }^{5}$, Munir Nayfeh ${ }^{6}$, and Ammar Nayfeh ${ }^{1}$ \\ ${ }^{1}$ Khalifa University, Abu Dhabi, 127788, UAE \\ ${ }^{2}$ College of Engineering and IT, University of Dubai, Dubai, UAE \\ ${ }^{3} N Y U A D$, Abu Dhabi UAE \\ ${ }^{4}$ University of Toronto, Toronto $\mathrm{Ca}$ \\ ${ }^{5}$ Cleveland Clinic Abu Dhabi, UAE \\ ${ }^{6}$ Department of Physics, University of Illinois, USA
}

Corresponding Author: Ammar Nayfeh

\begin{abstract}
Commercial polyethylene (PE) fiber-based masks are currently used as personal filters for protection against various microorganisms. Due to the coronavirus (SARS-CoV-2) pandemic of 2020, the use of masks has become the critical mechanism in reducing the spread. The PE mask filter uses a sieve (geometry) in a spider web fashion to filter out microorganisms using Van der Waals atomic forces. However, the non-geometrical part of the filtration process is not fully understood. In this work, we utilized luminescent ultra-small silicon nanoparticles, which are Si$\mathrm{H}$ or/and Si-OH terminated to examine how the filter operates at a chemical level. The particles were sprayed onto the fiber network by an atomizer and we used scanning electron microscopy (SEM), optical microscope and fluorescence spectroscopy under UV radiation. The images and measurements clearly showed that the Si nanoparticles bonded to the PE fiber network. The results were analyzed in terms of chemical bonding between Si nanoparticle and fiber. Our findings suggest that the PE fibers could act as a chemical filter via hydrogen or hydrolysis-based bonding or via Si-C bonding, which is complementary to their physical filtration ability via the geometric sieve process. Moreover, the results indicate that the filter would be effective against the novel coronavirus, not only by the geometrical sieve but can be additionally enhanced as a chemical filter by allowing the amine, carboxyl and hydroxyl function groups of the virus to attach to fibers via $\mathrm{C}-\mathrm{N}$ and $\mathrm{C}-\mathrm{O}$ bonds.
\end{abstract}




\section{Introduction}

In March 2020, the World Health Organization (WHO) declared COVID-19 a pandemic. The disease is caused by SARS-CoV-2 virus, which emerged in Wuhan, China in 2019 [1]. The virus is highly contagious and has a higher case fatality rate than other known respiratory viruses. The nature of transmission of the infection was not clear at the beginning and extreme precautions were in place when looking after patients with this infection. Similarly, the public and governments alike took different, often extreme, measures to halt the virus transmission. It is crucial to study the effectiveness of Personal Protective Equipment (PPE) including masks in preventing the transmission of the virus. [2-6]. Surgical masks are masks that are used in the healthcare setting to prevent the transmission of potentially pathogenic microorganisms from those who wear the masks to those who are at risk of contracting them by trapping the microorganisms that are shed into the respiratory secretions. Most masks are made by bonding nonwoven thermoplastic fabrics together, utilizing mechanical, heat or chemical methods. Spunbond and melt-blown are the two most popular of those methods. Both techniques can produce variable fabrics such as polyamide, polyester, polyethylene, polypropylene, and polyurethane [7-9]. However, polyethylene (PE) is the most common as it is relatively cheap with low melt viscosity and easily processed [10]. Epidemiologic studies showed that respiratory secretions droplets from infected individuals that are generated through coughing and sneezing are the main method of viral transmission. [11-17]. It is important to understand the interplay of geometric and chemical characteristics of the surgical masks in filtering SARS-CoV-2 virus. [18-21]. To date, to the best of our knowledge, there are no studies that examined how PE filter masks capture SARS-CoV-2 virus. Despite the interest in the use of PE filter masks to isolate the virus, there has been no studies that discern the basic principle of operation of the filter, and the interplay between sieve-based and the chemical/biological-based operation. In this work, we utilize for the first time luminescent ultra-small silicon nanoparticles to examine the filtering process of the polyethylene (PE) mask filters. We examine some of microscopic implications of defects in commercial PE masking filters. Silicon nanoparticles have several advantages. First, they are terminated by $\mathrm{Si}-\mathrm{H}$ or/and $\mathrm{Si}-\mathrm{OH}$ termination, which makes them chemically active through hydrogen bonding or hydrolysis-based bonding. Secondly, chemical attachment through covalent bonding is feasible since the hydrogen bond can be easily softened or cleaved via heating, irradiation, or oxidizers. Third, because they are ultrasmall $(\sim 3-\mathrm{nm})$ they are highly luminescent under $\mathrm{UV} /$ blue irradiation thus one can follow the path and landing through luminescence spectroscopy and microscopy. Fourth, because they are hydrophobic, clusters of diameters on the range of 100-300 nm 
across may form, which is similar to the virus size. Fifth, one may be able to use shifts in spectral distribution of the luminescence to discern the kind of bonding they make with the filter (physical vs. chemical, such as Si-C bonding). We used scanning electron microscopy (SEM) as well as optical microscopy to image the surface topography, bubble defects and the degree of roughness/randomness. The aggregation of silicon nanoparticles will be correlated with the defect distribution. The spectral distribution of the luminescence will be analyzed to discern the nature of attachment of the silicon nanoparticles to the fiber chemical vs. physical, namely of the defect cause changes in the molecular structures such as stripping of hydrogen and invitation of other active groups.

\section{Experimental Details}

\section{Si Nanoparticles}

The Si nanoparticles in this study are produced by a chemical etching of boron doped $100 \mathrm{Si}$ wafers [22]. We treated the wafer in a mixture of $\mathrm{HF}$ and hexachloroplatinc $\left(\mathrm{H}_{2} \mathrm{PtCl}_{16} \mathrm{H}_{20}\right)$ acid to deposit a platinum catalyst. The wafer is then etched briefly using $\mathrm{HF} / \mathrm{H}_{2} \mathrm{O}_{2} /$ methanol mixture. The wafer is then sonicated in a liquid of choice. Figure 1(a) gives the TEM image of a typical nanoparticle sample deposited on graphite grid; a histogram gives counts of 4, 56, 12, 9, and 2 at diameters of 1, 1.7, 2.2, 2.9, and $3.5 \mathrm{~nm}$. The first four produce wide emission bands at wavelengths of 410, 540, 570 and $600 \mathrm{~nm}$ respectively. Unlike the sharp emission from nanoparticles of direct-bandgap material, the wide emission by single sized-particles here is due to the fact that the precursor material, i.e., silicon is an indirect bandgap material. This gives a total of 11 particles with the smallest size (blue-green emitters) and a total of 72 particles of larger particles (red-orange emitters) with a ratio of $11: 72$ or $85 \%$ purity of the larger particles. In other words, the chemical procedure produces a soup of particles with red luminescent particles (size of $2.9 \mathrm{~nm}$ in diameter) dominating the distribution.

Figure 1b gives the emission spectrum of the particle colloid under incoherent UV light from a Xe lamp source at a wavelength of $355 \mathrm{~nm}$. It resolves two bands: a blue-green band and an orange-red band, with an intensity ratio of the blue to the red being 0.6 . The red-orange band covers the range of 540-720 $\mathrm{nm}$, with band head at $\sim 620 \mathrm{~nm}$. The weaker blue green band covers the range $\sim 400-480 \mathrm{~nm}$ corresponding to emission from the 1 and $1.67 \mathrm{~nm}$ diameter blue green emitting fraction of the particles seen in the TEM measurement. The quantum efficiency of emission of the red emitting particles is $\sim 30$ percent while that of the blue emitting particles is $\sim 60$ percent. The particles of sizes 1.7 and $1 \mathrm{~nm}$ emit 
radiation in the blue green part of the spectrum, while the 2.2, 2.9 and $3.5 \mathrm{~nm}$ emit in the red-orange range. The emission bands of all of the particles can be excited in the UV at wavelengths at $\sim$ or below350 nm, but their emission can also be induced (shows local resonance structures) when excited at 3.4, 2.64, 2.4 and $2.1 \mathrm{eV}$ respectively (Figure 1c). Those energies are the opening of their individual spatial quantum confinement bandgaps. Moreover, for excitation in the UV, the efficiency drops with the Stokes shift (energy difference between the excitation and emission photon energies). When examined the solution can be seen by the naked eye to be dominated by brightly red-orange luminescent under $365 \mathrm{~nm}$ irradiation as can be seen in the photoluminescent photo of the colloid given in Figure 1d. Figure 1d shows that when a soup of the particles solidifies on a silicon surface, same size particles assemble (crystalize) together, showing their distinct luminescence colors [23]
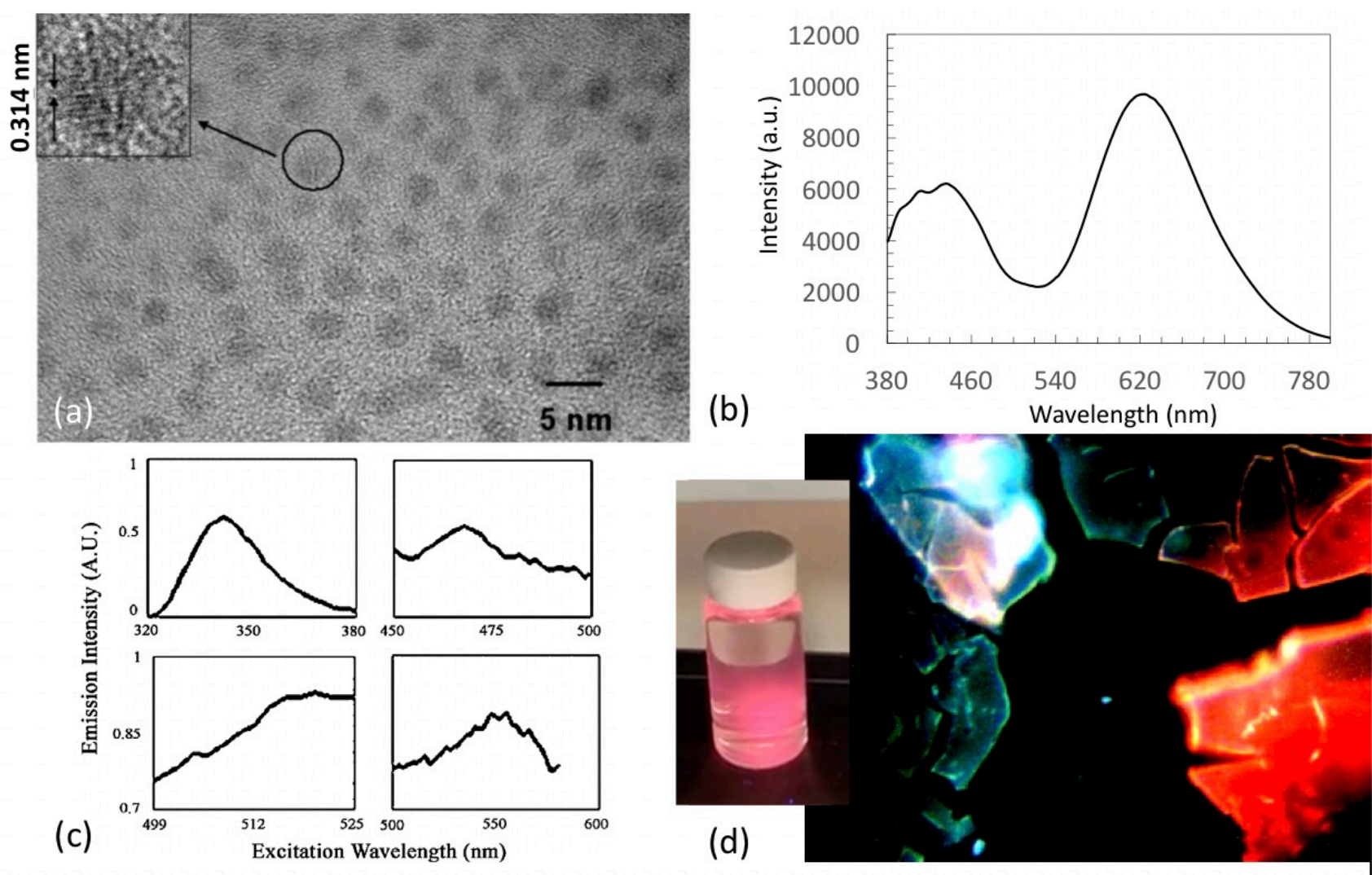

Figure 1. (a)Transmission electron microscopic image of individual Si NPs and (b) Emission spectrum of the Si NPs colloid showing a red-orange band and a smaller blue-green band under irradiation at $355 \mathrm{~nm}$ UV radiation. (c) emission spectra of $1,1.67,2.2$, and $2.9 \mathrm{~nm}$ particles with excitation at their respective confinement 
bandgaps [23]. (d) The Si NPs solution can be seen to be dominated by brightly red-orange luminescence under $365 \mathrm{~nm}$ irradiation and the self-assembly on a surface of same size (emitting same band) nanoparticles

\section{Chemical structure of the masks}

The masks used are commercial products. The interaction of nanoparticles with microporous membranes or networks of polyethylene (PE) plastic fibers has been receiving concerted interest due to the ongoing SARS-CoV-2 challenge. The elementary structure of PE membranes is a repeating linear molecular structure or chain, the building unit of which is the hydrocarbon $-\mathrm{CH}_{2}-\mathrm{CH}_{2}$. The molecules can make solids in form of pellets, sheets, fibers, filaments. Those structures have found applications in a myriad of devices, an urgent one of which is a protective gear (masking) against virus entry into the lung system. They are also widely used in laboratory applications for solid-liquid filtration/separation, medical devices, drug delivery devices, automotive industry, water filtration systems, and medical and surgical applications.

On a microscopic level, the importance of this material stems from the fact that the PE molecule is inert with strong molecular bonds and stable chemical resistance, as well as having a low drug or protein adsorption. In addition, it is nonpolar, hydrophilic and hydrophobic and self-sealing in water. It is a saturated (single bonds) hydrocarbon structure. As such, the chain macromolecules do not covalently link to each other but can associate with each other through weak atom-atom forces to make variety of material structures. Because of their inertness, they do not easily stick to surfaces, and as such they cannot be imprinted or bonded with adhesives without pretreatment. To achieve high strength joints, it is customary to use plastic welding. Moreover, their symmetric molecular structure in a solid form makes them show crystallinity and higher density albeit partial. As to the gas permeability in their solid form, polar gasses experience lower permeability than oxygen, carbon dioxide and flavorings, which can pass it easily. In their fiber form, they can be made in cloth configuration and used as gas filters. Filters based on inert material, such as PE operate on mechanical or geometrical principles (sieve effect), which is one most commonly applied in air filters. The principle of the effect is that the particle to be filtered is larger than the gap between the fibers and therefore the filter traps it without interfering with the chemistry of the particles. To prevent build-up, the filter must be cleaned regularly or discarded. Replacement is only required when the filter can no longer release all the dirt that it holds.

The types of masked used in this work are highlighted in Table 1. They were compared to the lack of a mask barrier (no mask control) 
Table 1. Masks' type, material and application that were used in this work.

\begin{tabular}{ccc}
\hline Type & Brand & Material \\
\hline $\begin{array}{c}\text { Cleanroom } \\
\text { Mask }\end{array}$ & $\begin{array}{c}\text { KIMTECH PURE } \\
\text { M3 Face Mask }\end{array}$ & Polypropylene and BiCoSof \\
Surgical Mask & Dynarex 2201 & $\begin{array}{c}\text { Polypropylene melt-blown } \\
\text { non-woven fabric }\end{array}$ \\
\hline FFP2 Mask & $3 \mathrm{M}$ & $\begin{array}{c}\text { Polypropylene and ethylene- } \\
\text { vinyl acetate (EVA) }\end{array}$ \\
\hline N95 Mask & $3 \mathrm{M}$ & $\begin{array}{c}\text { Filter - Polypropylene Shell - } \\
\text { Polyester }\end{array}$ \\
\hline
\end{tabular}

\section{Spray Deposition}

We first studied the effect of aerosolized deposition of Si nano. The aerosolized droplets pattern from the spray bottle was first optimized_by spraying a Si wafer under UV irradiation as shown in Figure 2. 


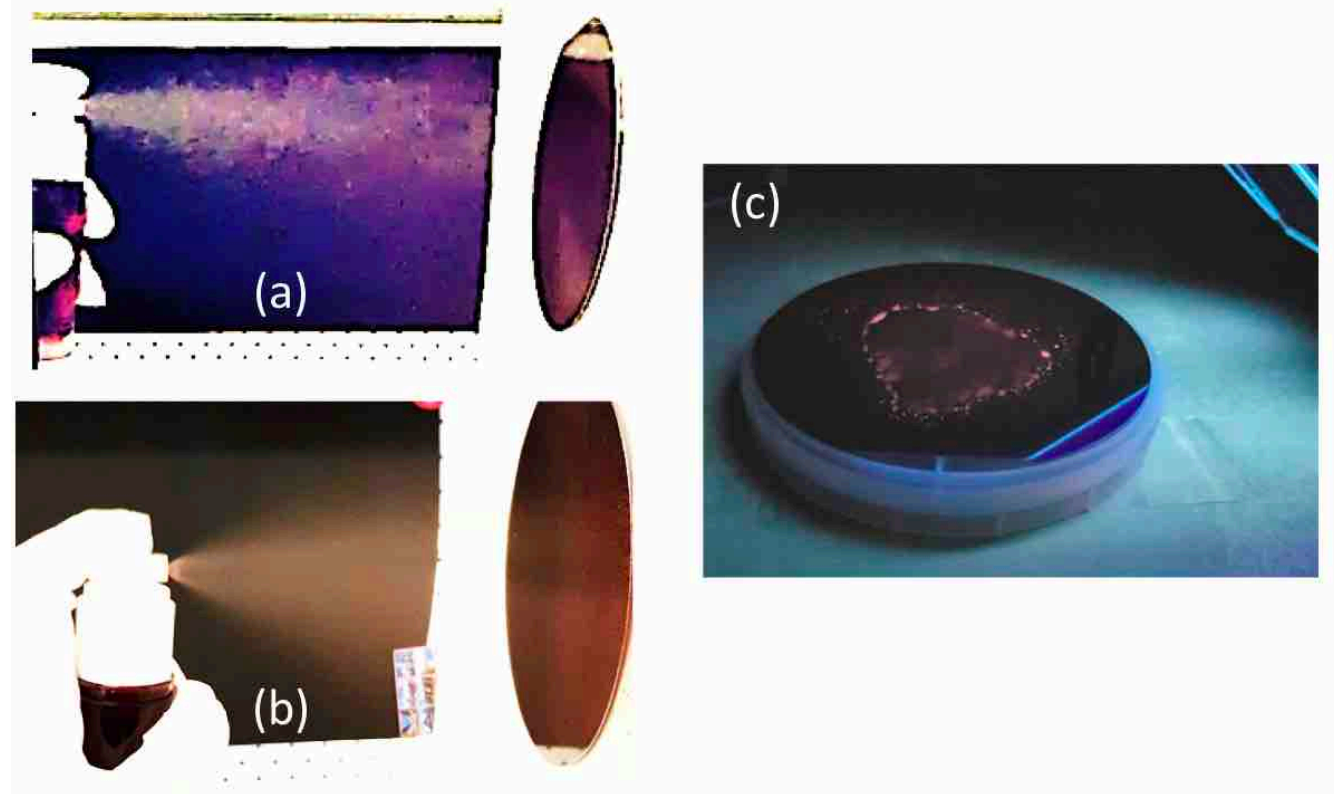

Figure 2. (a) and (b) Optimizing the spray coating pattern on a Si wafer. The spray cloud under exposure of UV radiation with a wavelength of $365 \mathrm{~nm}$ (c) An example of a Si wafer under UV irradiation after spraying the Si NPs.

Using the spray deposition and the Si nano, the mask filter process will be studied. We used a spray bottle filled with Si NPs dispersed in isopropyl alcohol (IPA) to simulate a cloud of droplets. The 50 $\mathrm{mL}$ spray bottle used, shown in Figure 3(b), is a commercially available spray device capable of delivering 200 sprays per bottle with $250 \mu \mathrm{L}$ of fluid per actuation. We chose to use a pump spray dispenser over propellant spray dispenser. To prepare a suspension of Si-NPs in IPA, $5 \mathrm{~mL}$ of the $\mathrm{Si}$ NPs dispersion was added to $10 \mathrm{~mL}$ of IPA, sonicated for two hours to ensure a homogenous solution. Before testing, spray bottle nozzles were adjusted to produce cloud and jet-propelled droplets that match the visual architecture of droplet formation described in [24, 25].

Typical pump spray dispensers produce various liquid droplet dynamic phases occurring within a single spray [26], which reproduces results in a wide arrange of droplet sizes with a distribution range between 20 and $900 \mu \mathrm{m}$ [27]. For comparison, the size of droplets in a typical sneeze range between 40 and 900 $\mu \mathrm{m}$ [28]. The spray bottle ejects fluid with pressures that can reach a pressure of $10 \mathrm{psi}$ to create a short burst of microdroplets. In context, the pressure during a sneeze is 1 psi in the trachea and 2.6 psi in the mouth [29-31]. In this work, one spray pass ejects $250 \mu \mathrm{L}$ of fluid per spray, therefore four consecutive spray passes, delivered at $1 \mathrm{pump} / \mathrm{second}$, constitute an exposure of $1 \mathrm{ml}$ of fluid in $4 \mathrm{~s}$. 
Since the nanoparticle colloid has a distribution of nanoparticle sizes, we expect the nanoparticles, if dried, to cluster with similar particles tending to self-assemble. Thus, the smaller blue-green particles may go together while the larger nanoparticles are expected to go together. When the particles in a colloid are sprayed onto a substrate using a jet/nozzle using differential pressure, they form droplets that encapsulate particles of similar size. In the present atomization, we expect approximately two components: red-orange emitting droplets and blue-green emitting droplets. The volatile liquid may evaporate in flight to a large degree, allowing the particles to land on the substrate.

\section{Setup with Mask}

To simulate the function of mask barriers, we placed selected masks framed between the spray bottle and $3 \times 3 \mathrm{~cm}$ Si wafer piece mounted over a foam surface and above the table surface, through which droplets were sprayed as depicted in Figure 3(a,b,c). The dispenser nozzle tip was placed perpendicular to the masks at a horizontal distance of $10 \mathrm{~cm}$ mask which is around half the distance between the nostrils and vocal cords $(\sim 15-20 \mathrm{~cm})$ [32], or the lip-to-carina distance $\sim 20-25 \mathrm{~cm}$ [33] in humans. Quantification represents droplets that pass through the masks and that land on Si pieces. All testing conditions were carried out at constant ambient conditions. 

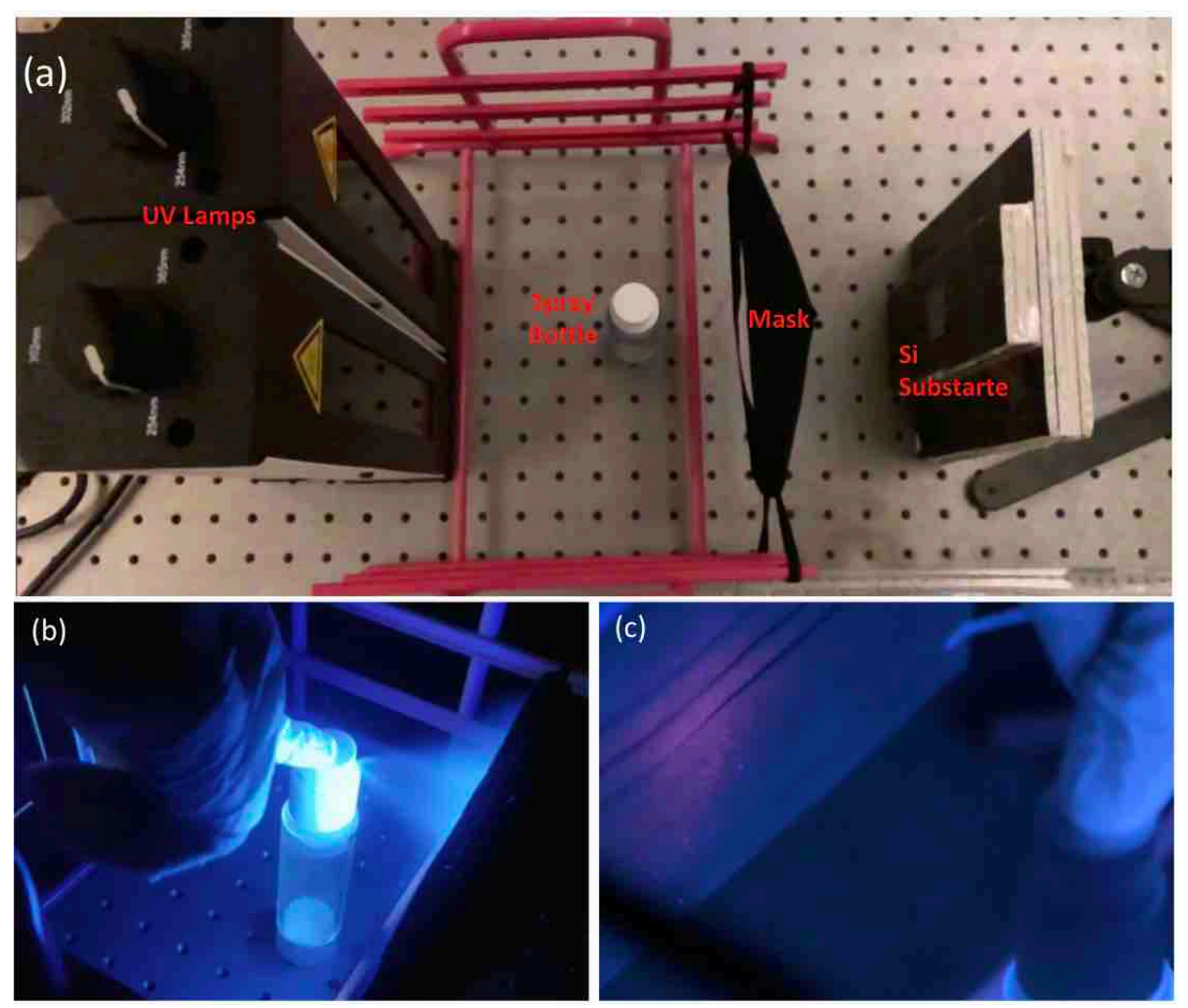

Figure 3. (a) Top view of the setup used to spray the masks (b) Spray pattern from the spray bottle (c) Spraying the mask under the UV lamp

\section{Results}

The mask fibers were studied using several diagnostic procedures including optical, luminescent and electron spectroscopy. It was imaged using scanning electron microscopy (SEM), fluorescence and optical microscopy, and luminescent spectroscopy. Diagnostics were made while the particles were in the colloid and after landing on the fibers.

\section{Electronic imaging}

Since the fibers are non-conducting the resolution of SEM is limited. Standard use of deposition of a thin film of gold palladium on the fibers to enhance the resolution was not used to avoid quenching of the luminescence of the nanoparticles. The topographical characteristics of these samples were not controlled. Scanning electron microscopy (SEM) images shown in Figure 4 show that the surface topography and roughness are random. One can see that the fibers have a diameter of $\sim 25 \mu \mathrm{m}$. The average characteristic size of the pores in the filter can be in the sub micrometer range. Also, one 
observes bubble defects of an average diameter of $100 \mu \mathrm{m}$. One can see that during extrusion the fiber avoids the bubbles causing, bending, twisting, flattening, cracking, and increased roughness as well as piling up at the edges. In some cases, it causes interruption of the flow of the fibers, ending at the edge of bubbles. Such distortion and strain may induce bond cleavage hence a variety of hydrogen charge states on the surface. Broken hydrogen bonds may pick up environmental oxygen atoms. Figure 4 shows SEM images of the surgical mask where the Si NPs clusters trapped between the mask fibers. These clusters are not observed in uncoated masks as exhibited in Figure 4
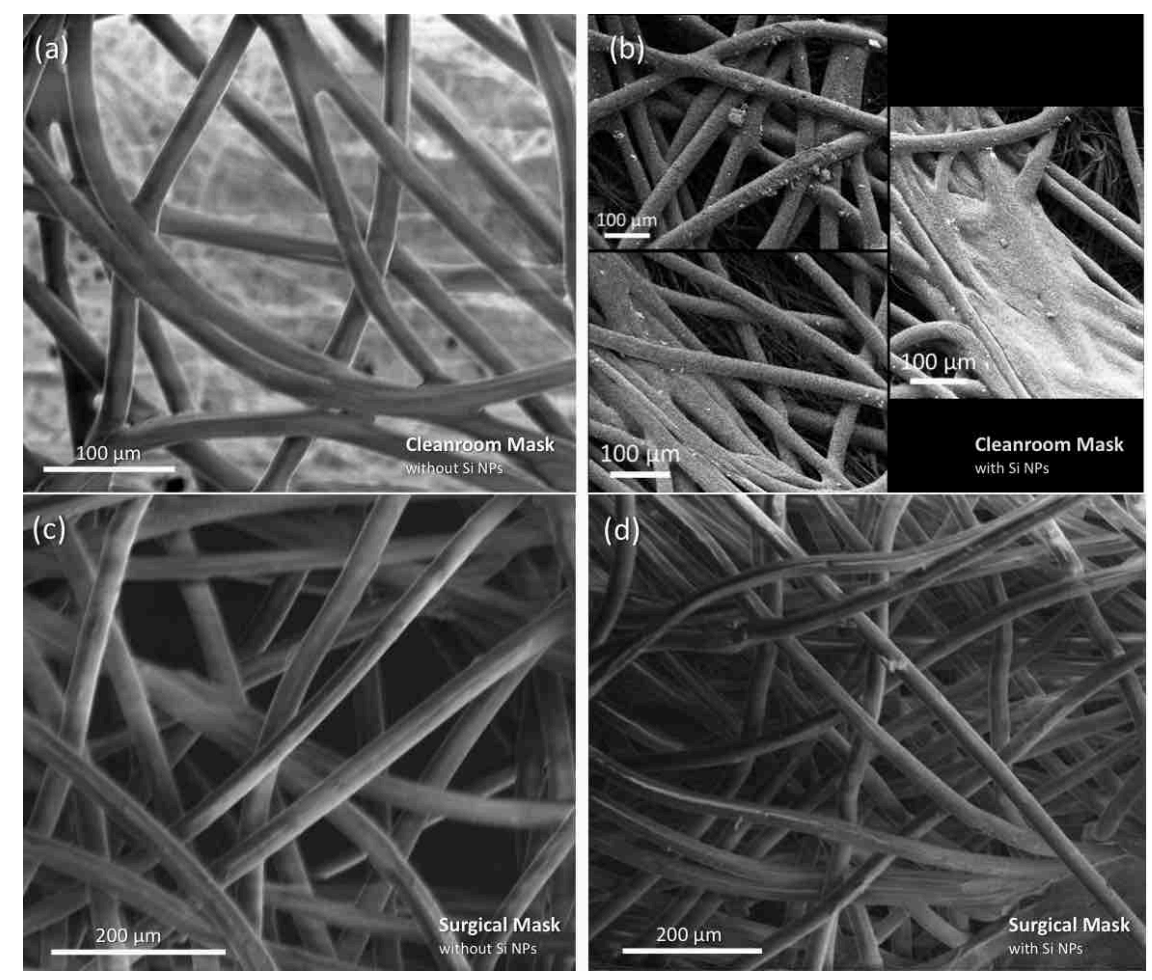

Figure 4 Scanning electron microscope image of the (a-b) cleanroom and (c-d) surgical mask with and without Si nano particles.

\section{Luminescent imaging}

Luminescence studies included imaging, microscopy, and spectroscopy. Figure 5 shows luminescent images of two filters taken under UV excitation at $365 \mathrm{~nm}$ after they have been removed and dried under ambient conditions. Figures 5(a) and 5(b) show luminescent photos of the coated surgical mask and of a control mask, respectively, both taken under excitation at a wavelength of $365 \mathrm{~nm}$. The blue color is background caused by scattering of incident ultraviolet (UV). Figure 5(b) which gives the luminescence from the coated mask and shows the red/orange luminescent as very bright, indicating that the quantum 
efficiency of the particles has changed little if any upon surface contact. Imaging shows that the coating is not fully uniform, but it is comprehensive.
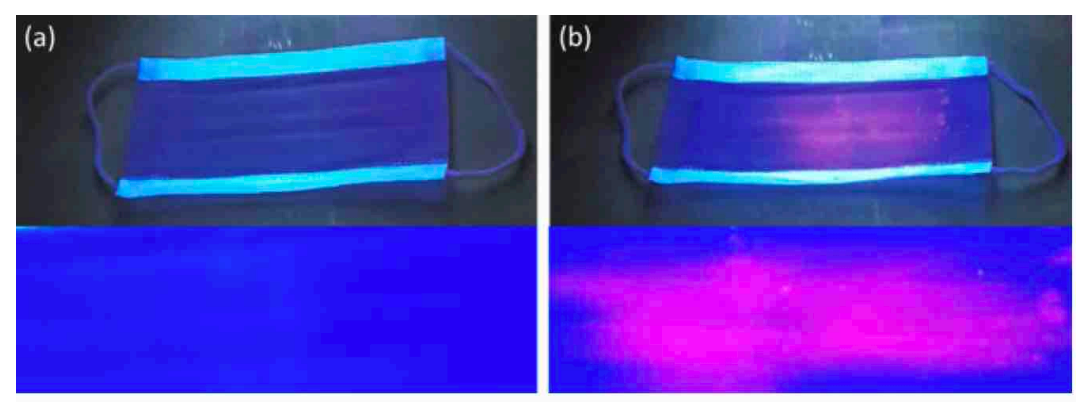

Figure 5 Surgical mask under UV (a) before spraying and (b) after spraying the Si NPs

\section{Luminescent microscopy}

Figure 6 gives luminescence image of a section of the cleanroom mask using luminescence microscopy. Instead of using a florescence microscope where the UV light is built in and focused on a small section of the fiber mask $(1 \mathrm{~mm})$ we used an optical microscope while illuminated with an external UV source covering a much larger area of the mask. Figure 6a-b gives images before and after spraying of the nanoparticles, respectively. We can see strong luminescence emanating from the fibers. Figure 6c-d shows the same effect on a surgical and N95 masks, respectively. The strong luminescence was exclusive to coated masks and is not observed in uncoated masks. 

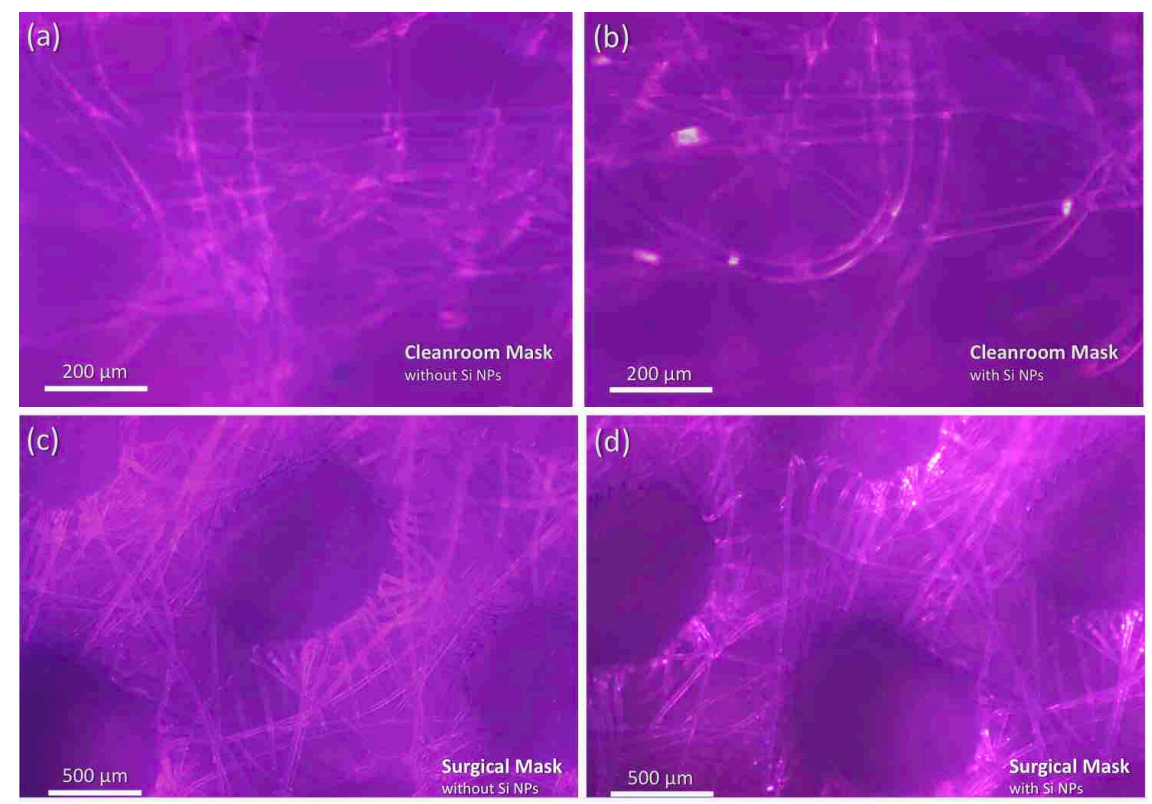

Figure 6 An optical image of the (a-b) surgical and (c-d) cleanroom mask under UV with and without Si nanoparticles.

\section{Photoluminescence spectroscopy}

The nanoparticles on the masks were examined using luminescence spectroscopy to determine the emission characteristics and the effect of the mask molecules on the particles and whether the particles are physically or chemically attached through some kind of bonding. The light source used is a continuous output from a Xe arc lamp, which delivers $150 \mathrm{~W}$. An integrated, selectable low-pressure mercury lamp light source is used for validation of this emission, while a radio-photometer system using monochromatic light is used to monitor the intensity output of the Xe lamp. The monochromatic uses a holographic concave grating in modified Rowland mount. The wavelength range using the zero order of the grating can be scanned over a $200-850 \mathrm{~nm}$ range. The detector used in the excitation arm is a silicon photo diode (PD). On the emission arm, the system uses zero order covering the range $200-750 \mathrm{~nm}$. The emission detector is a liquid nitrogen cooled photomultiplier (PMT). The system provides a very low stray light level and a dynamic range of up to 6.5 orders of magnitude. The wavelength resolution is $1.0 \mathrm{~nm}$ for both excitation and emission arms. This high spectral resolution of $1.0 \mathrm{~nm}$ is augmented with an automatic band-pass filter module for exclusion of higher-order diffraction.

Figure 7(bottom) gives the intensity of luminescence from the coated mask as a function of wavelength. The excitation wavelength is fixed at $355 \mathrm{~nm}$ while the emission arm is scanned over the range 370-760 nm. For easy comparison, we give in Figure 7(top) the spectrum for a colloid sample as was shown in 
Figure 1b. As discussed above the spectrum shows a red-orange and a blue-green band. We make several observations. First, we see a relative drop in the red to blue ratio upon deposition on the mask. After spraying, the red band intensity becomes smaller than that of the blue band. Second, upon deposition we see a drop in the red wing of the red band relative to its blue wing, which causes an apparent shift in the center of gravity of the band towards the blue by nearly $35 \mathrm{~nm}$. Third upon deposition we see no change in the structure of the blue band hence no shift. On the average, the nanoparticle coating appears to have a pink tint compared to the red tint from the colloid indicating the increase of the blue band strength relative to the red band.

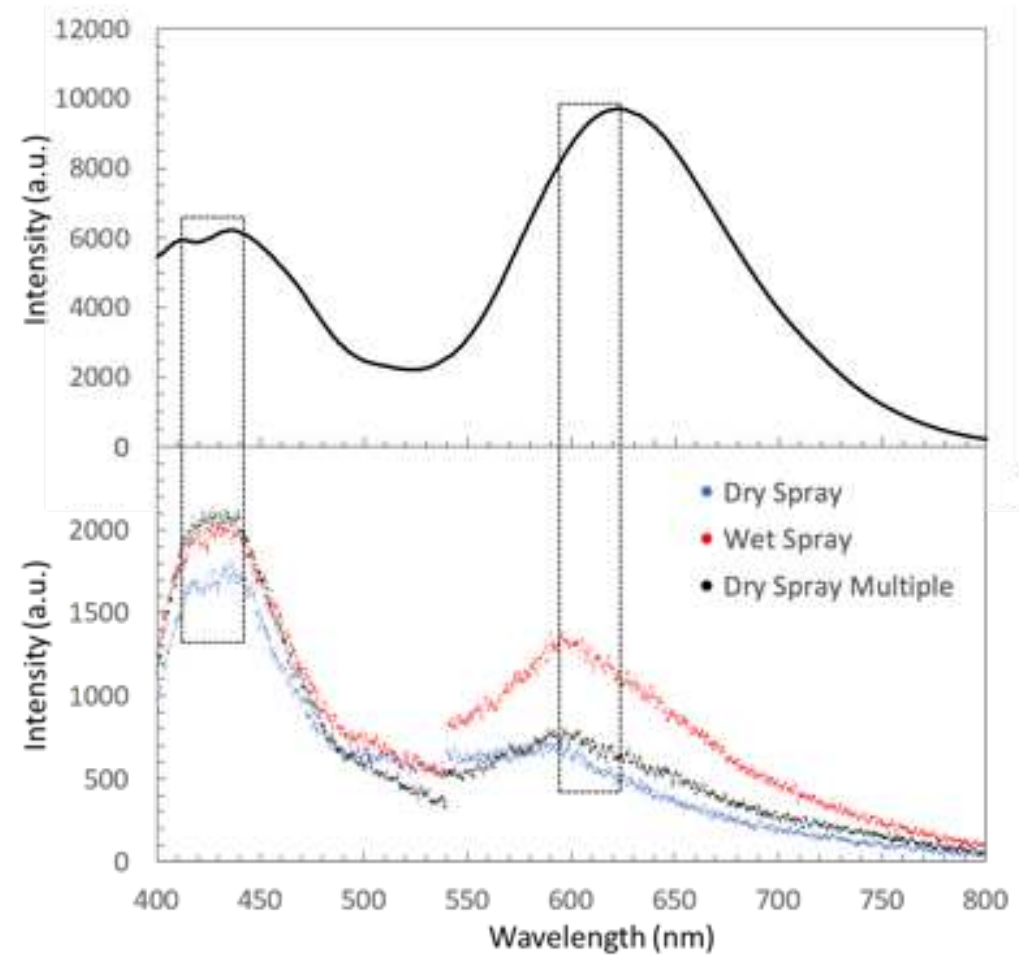

Figure 7 Luminescence as a function of wavelength on (top) Si-NPs solution and (bottom) mask with Si-NPs. The excitation wavelength is fixed at $355 \mathrm{~nm}$ while the emission arm is scanned over the range 370-760 nm. The discontinuity in the spectra at is due to a filter change.

It is interesting to isolate the red luminescence band by exciting at $488 \mathrm{~nm}$. At this wavelength, the photon energy is below the excitation energy of the blue band. Figure 8 gives the corresponding emission as a function of wavelength. FFP2 is a surgical mask made of three layers: a filter layer made of a melt-blown polymer, most commonly polypropylene placed between two non-woven fabric layers. The melt-blown material is the material preventing the microbes from exiting the mask. _The position of the measured red-orange band is in reasonable agreement with the band excited using UV radiation at $350 \mathbf{~ n m}$ as shown in Figure7. The corresponding emission for a stretchy nylon mask (CR mask) is also 
given in Figure 7. The mask is a non-surgical mask, which is made from stretch nylon fabric. The spectrum is found to be blue shifted slightly relative to the polymer mask.

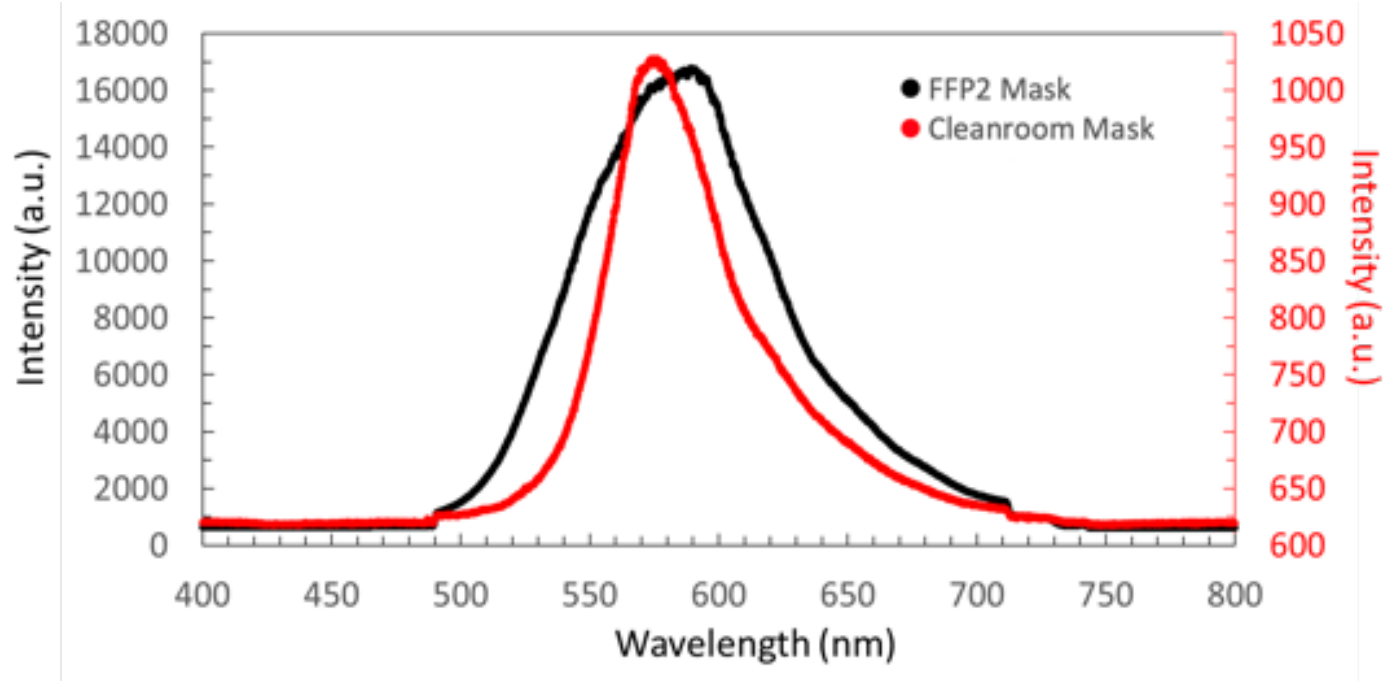

Figure 8 The PL spectra of coated FFP2 Mask and Cleanroom Mask at 488nm

\section{Analysis and discussion}

First, the above results show that there is a change in the red part of the emission spectrum of the nanoparticles when they are deposited on the mask. Such change in the spectral distribution may shed some information on the nature of attachment of the silicon nanoparticles to the fibers. For instance, the spectral change suggests that it is more probable that the deposition is of a chemical nature than of a physical nature. For the particles to chemically attach, there must be some active sites (defects) since the PE structure is inert. This may point to the presence of defect sites on which the nanoparticles may be attached. Defects may be created by striping some of the inert hydrogen termination off the fibers, making them active sites, which may also be followed with substitution by active groups. This effectively result in changes in the molecular structures of the fibers.

Defects may arise during manufacturing or due to effect of external agents. External agents can affect $\mathrm{PE}$ in general and fibers in particular. Weather of mechanical, chemical or radiation origin they are very important for their use in devices. First, the manufacturing process of commercial products is not fully controlled at the microscopic or molecular level. Bubbling during the extrusion process may introduce high mechanical strain or interruptions, which may introduce microscopic defects as was shown in Figure 4. Such defects may include those due to severing hydrogen and creation of dangling bonds, 
which makes the structure amenable to environmental attack. Although PE fibers in general_are not attacked by strong acids or strong bases and are also resistant to gentle oxidants and reducing agents, interaction with strong peroxides can soften or severe hydrogen termination. Light can also create defects in PE. For instance, hydrogen termination may be softened or severed by exposure to strong light / UV. In fact, PE is known to become brittle when exposed to sunlight due to cleavage of hydrogen and would crumble into small pieces. To alleviate this feature, carbon black is usually used to intercept UV and as such act as a UV stabilizer. The dangling bonds due to cleavage can also be easily saturated with oxygen or hydroxyl groups, resulting from environmental interactions, which may compromise the inert nature of the filter but allow chemical attachment to other agents.

We now discuss some plausible processes that may proceed at defect sites between the fibers as well as between external species and the fibers. An interaction of defect on a certain fiber can occur with another nearby fiber such that the two fibers are crosslinked through C-C bonding as shown in Figure 9.
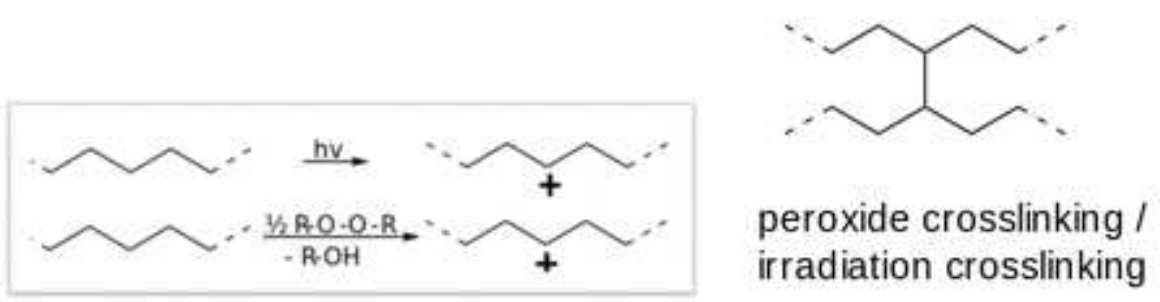

Figure 9. Under UV irradiation or action of hydrogen peroxide, the softened of the hydrogen bond in the polyethylene fiber causing the two fibers to crosslink through C-C bonding.

The present results have shown Si nanoparticles bond to the fibers of the various masks examined, acting as an effective barrier. We now discuss a plausible process for the bonding. Silicon nanoparticles may be partially oxidized resulting in termination by the $\mathrm{OH}$ function group ( $\mathrm{Si}-\mathrm{OH}$ ) without compromising their luminescence. In this case, hydrogen bonding takes place with the fiber when the system is dried. Also, the $\mathrm{Si}-\mathrm{OH}$ of the nanoparticle can react with the $\mathrm{C}-\mathrm{H}$ bond of the fiber to form the $\mathrm{Si}-\mathrm{C}$ bond. In addition, as mentioned above, under UV irradiation the hydrogen bond is softened or cleaved in both the nanoparticles and the polyethylene fiber. A similar interaction allows the bare silicon and the bare carbon atoms in PE to form a Si-C bond as shown in Figure 10(a). The same nanoparticle could interact with another PE chain, essentially acting as a secondary cross linker as shown in Figure 10(b). The formation of the Si-C bond has been examined previously in 1-nm particles using first principle atomistic simulations and calculations. It was shown to increase the band gap of the particle because a $\mathrm{C}$ atom 
tends to repel the electrons to the inside of the particle. This may cause a shift in the center of the nanoparticle emission band towards the blue.
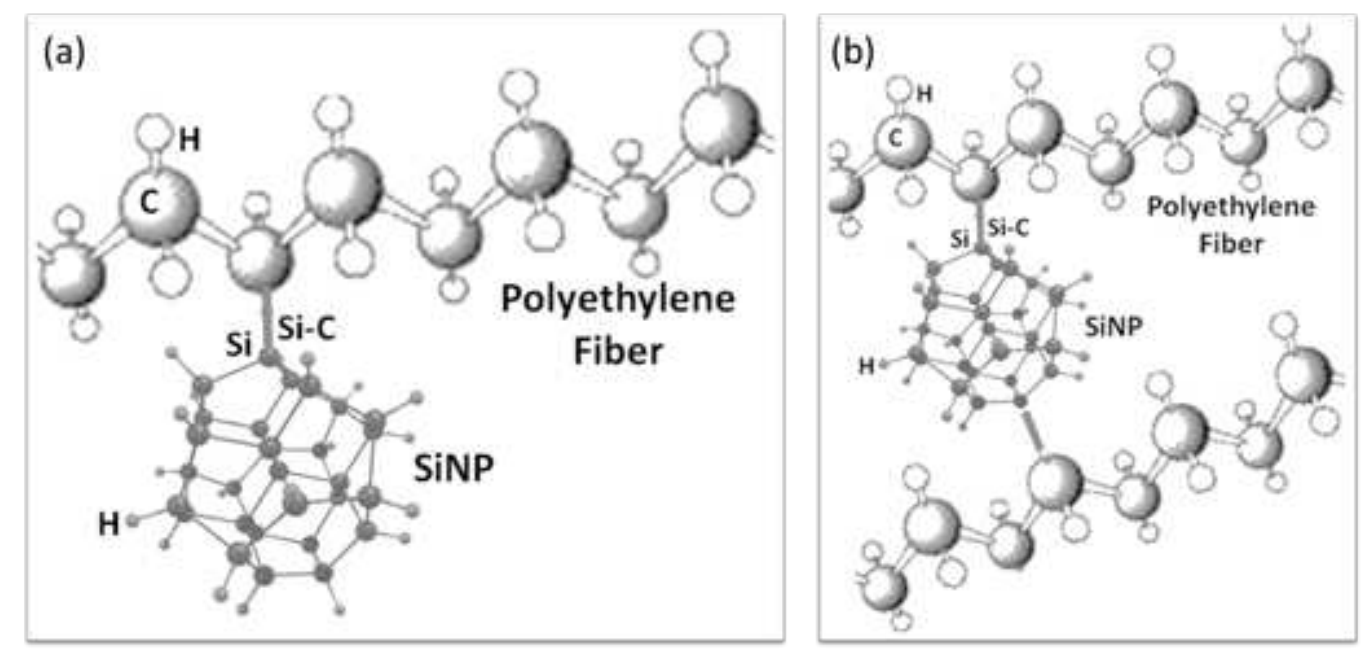

Figure 10. (a) The Si-C bond form between the bare silicon and the carbon atoms in PE. (b) The Si NP interacting with multiple PE chain while the nanoparticle acts a secondary cross linker.

The above spectral results given in Figure 7 show that the emission intensity ratio of the blue-green band and the red-orange band changes upon deposition on the mask. In the colloid, the intensity ratio of the blue to the red is 0.6 . On the mask the ratio is 1.8 . Since smaller nanoparticles, are responsible for the blue band, whereas larger nanoparticles (red emitting) are responsible for the red-orange band, one can conclude that the number ratio changes upon deposition. This may indicate that the droplet having smallest nanoparticle clusters fly longer in the air compared to those having the larger particles. It is also plausible that the change in the ratio is due to variations in the charge exchange processes between the chemically attached species, due to variations in the energy scales in the different sized particles. The confinement bandgaps of the blue and green emitting particles are 3.4 and $2.64 \mathrm{eV}$, compared to 2.4 and $2.1 \mathrm{eV}$ for the orange and red emitting particles respectively. One effect that one can mention is that a chemical attachment of the red emitting particles may make them more susceptible to luminescence quenching and reduction of quantum efficiency.

The results may have implication on the filtering ability against SARS-CoV-2. Based on this, the ability of the fiber to filter out corona virus is interesting to address. The result shows the ability of the PE to chemically bond to Si-H or Si-OH. UV or light exposure can be used to photocatalyze binding of the nanoparticles to the fibers. Filtering SARS-CoV-2 presents specific active chemistry and challenges. The protein molecules that constitute the spike protein that facilitate the entry of the virus into human 
cells is mostly amino acids having amine (NH2) and carboxyl (COOH) active functional groups. The above reported results using the silicon nanoparticles may suggest that the fiber can also be an effective SARS-CoV-2 filter not only by the geometrical sieve process but also as a chemical filter by allowing the amine, carboxyl and hydroxyl function groups of the virus to attach to fibers. Figure 11 shows a plausible mechanism for PE to attach chemically to the amine and the carboxyl groups in the virus via the formation of C-O and C-N covalent bonds. This is indicative of the virus being drawn.

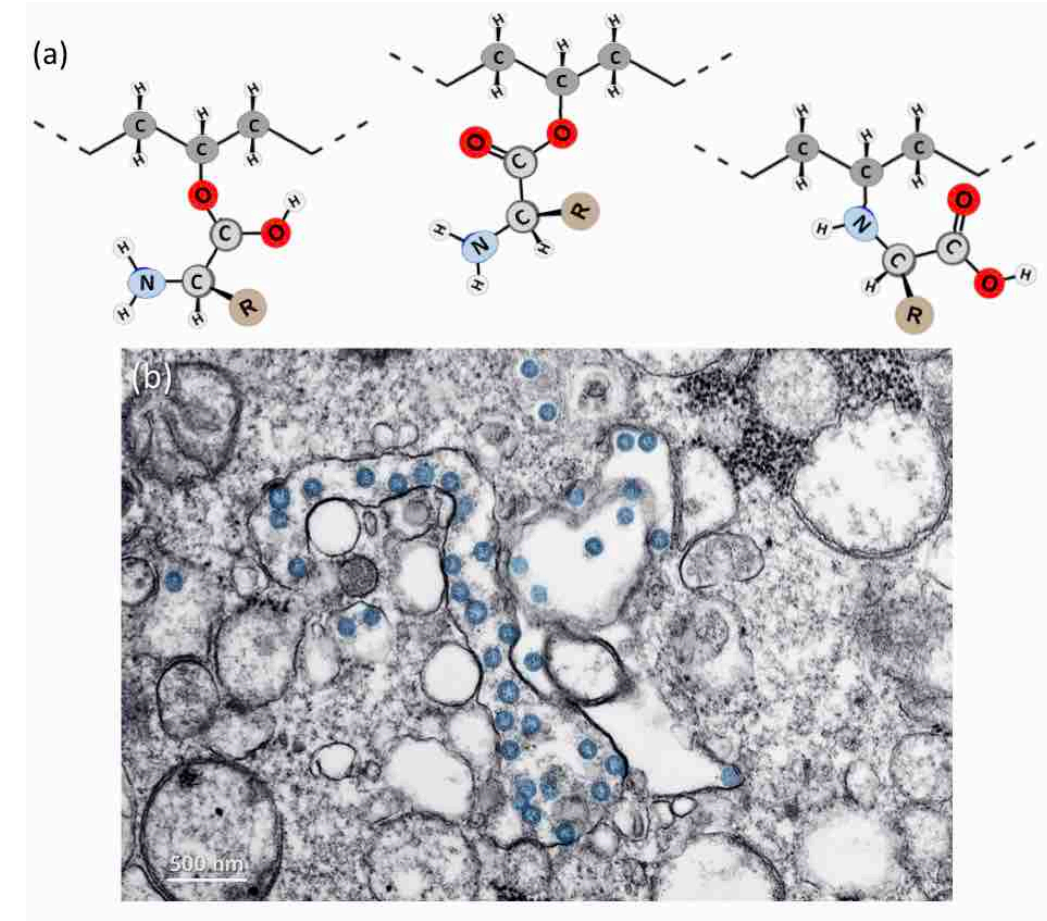

Figure 11. (a) The PE chemical attachments via the covalent bonds C-O and C-N (b) TEM image of COVID-19 virus. The spherical viral particles, colorized blue.

In addition, the protocols to disrupt the virus in human dwelling including the anti-virus action of oxidizers $\left(\mathrm{H}_{2} \mathrm{O}_{2}\right)$, UV, or alcohol may also be useful in activating the filters against the virus. Those agents tend to break many of the weak linkages, or bonds (e.g., hydrogen bonds) (denaturation), within the protein molecules that constitute the casing or wall as well as the entry mechanism of the virus into cells. Such action results in a looser, more random structure; most of which are insoluble, rendering it ineffective (destroyed). Adding water to alcohol catalyzes the breakage of bonds hence the destruction of the virus. Hydrogen peroxide, a strong oxidizer produces hydroxyl free radicals that attack membrane lipids, DNA, and other essential cell components in a virus. Thus, those chemical agents are also expected to affect the filter by allowing PE chains to crosslink with each other using strong covalent 
bonding. As discussed above, peroxide, UV and electron irradiation can soften or break hydrogen bonds in PE such that it gets activated to cross-link chains of PE through the formation of C-C bonds. Moreover, it activates attachment of $\mathrm{PE}$ to the amine $(\mathrm{NH} 2)$ or carboxyl $(\mathrm{COOH})$ groups of the spike proteins of the SARS-CoV-2.

\section{Conclusions}

We utilized droplets encapsulating luminescent ultra-small silicon nanoparticles to examine how commercial polyethylene (PE) fiber-based masks work as personal filters for protection against various microorganisms. The particles have different sizes, wavelength (color) of emission, as well as $\mathrm{Si}-\mathrm{H}$ or/and $\mathrm{Si}-\mathrm{OH}$ termination. The nanoparticles were sprayed onto the fiber network by an atomizer placed at a certain distance and UV-induced fluorescence was used to observe their flight from the atomizer source to the mask target. The studies show that smaller nanoparticles are more efficient at reaching the target, and that the PE fibers act as a chemical filtering device via hydrogen or hydrolysis-based bonding or via Si-C bonding in addition to the geometrical sieve filtering. Moreover, the results indicate that the filter would be effective against the novel coronavirus, not only as a geometrical sieve filter but additionally as an enhanced chemical/bio filter via attachment of the amine, carboxyl and hydroxyl function groups of the virus to fibers via $\mathrm{C}-\mathrm{N}$ and $\mathrm{C}-\mathrm{O}$ bonds.

\section{Acknowledgement}

This publication is based upon work supported by the Khalifa University of Science and Technology under Award No. CRPA-2020-002. 


\section{References}

[1] Timeline of WHO's response to COVID-19. Accessed July 6, 2020. https://www.who.int/newsroom/detail/29-06-2020-covidtimeline

[2] Visualizing the effectiveness of face masks in obstructing respiratory jets https://doi.org/10.1063/5.0016018

[3] Face Mask Use and Control of Respiratory Virus Transmission in Households https://dx.doi.org/10.3201/eid1502.081167

[4] A rapid systematic review of the efficacy of face masks and respirators against coronaviruses and other respiratory transmissible viruses for the community, healthcare workers and sick patients https://doi.org/10.1016/j.ijnurstu.2020.103629

[5] A schlieren optical study of the human cough with and without wearing masks for aerosol infection control https://doi.org/10.1098/rsif.2009.0295.focus

[6] Rational use of face masks in the COVID-19 pandemic https://doi.org/10.3201/eid2605.190994

[7] Lim H. A review of spun bond process. Journal of Textile and Apparel, Technology and Management. 2010;6:1-13. https://doi.org/10.15406/jteft.2017.01.00023

[8] Midha V., Dakuri A. Spun bonding technology and fabric properties: a review. Journal of Textile Engineering \& Fashion Technology. 2017;1(4) https://doi.org/10.15406/jteft.2017.01.00023.

[9] Dutton K. Overview and analysis of the meltblown process and parameters. Journal of Textile and Apparel, Technology and Management. 2008;6(1)

[10] Henneberry B. How surgical masks are made. https://www.thomasnet.com/articles/other/howsurgical-masks-are-made/

[11] The efficacy of standard surgical face masks: An investigation using "tracer particles https://doi.org/10.1097/00003086-198005000-00024

[12] A quantitative assessment of the efficacy of surgical and N95 masks to filter influenza virus in patients with acute influenza infection https://doi.org/10.1086/600041

[13] Respiratory virus shedding in exhaled breath and efficacy of face masks https://doi.org/10.1038/s41591-020-0843-2

[14] Assessment of a respiratory face mask for capturing air pollutants and pathogens including human influenza and rhinoviruses https://doi.org/10.21037/jtd.2018.03.103

[15] Simple respiratory protection-Evaluation of the filtration performance of cloth masks and common fabric materials against 20-1000 nm size particles https://doi.org/10.1093/annhyg/meq044

[16] Effectiveness of surgical and cotton masks in blocking SARS-CoV-2: A controlled comparison in 4 patients https://doi.org/10.7326/m20-1342

[17] Aerosol filtration efficiency of common fabrics used in respiratory cloth masks https://doi.org/10.1021/acsnano.0c03252

[18] Face Masks in the New COVID-19 Normal: Materials, Testing, and Perspectives https://doi.org/10.34133/2020/7286735

[19] Electrospun Nanofibers-Based Face Masks https://doi.org/10.1007/s42765-020-00049-5

[20] Reusability Comparison of Melt-Blown vs Nanofiber Face Mask Filters for Use in the Coronavirus Pandemic https://dx.doi.org/10.1021/acsanm.0c01562

[21] Filtration Efficiencies of Nanoscale Aerosol by Cloth Mask Materials Used to Slow the Spread of SARS-CoV-2 https://dx.doi.org/10.1021/acsnano.0c05025

[22] M. H. Nayfeh, Fundamentals and applications of nano silicon in plasmonics and fullerenes: current and future trends Elsevier Publishing, (2018)

[23] M. H. Nayfeh and L. Mitas, in Nanosilicon, V. Kumar, ed. Elsevier, 1 (2007) 
[24] Bourouiba L. Images in clinical medicine. A sneeze. N Engl J Med. (2016) 375:e15. 10.1056/NEJMicm1501197

[25] Size distribution and sites of origin of droplets expelled from the human respiratory tract during expiratory activities https://doi.org/10.1016/j.jaerosci.2008.11.002

[26] Rodriguez-Palacios A, Conger M, Cominelli F. Nonmedical masks in public for respiratory pandemics: droplet retention by two-layer textile barrier fully protects germ-free mice from bacteria in droplets. bioRxiv [preprint]. (2020). 10.1101/2020.04.06.028688

[27] Kooij S, Sijs R, Denn M, Villermaux E, Bonn D. What determines the drop size in sprays? Phys Rev. (2018) 8:031019 10.1103/PhysRevX.8.031019

[28] Han ZY, Weng WG, Huang QY. Characterizations of particle size distribution of the droplets exhaled by sneeze. J R Soc Interface. (2013) 10:20130560. 10.1098/rsif.2013.0560

[29] Burke W. Why do we sneeze? Med Hypotheses. (2012) 78:502-4. 10.1016/j.mehy.2012.01.017

[30] Simplified models for exhaled airflow from a cough with the mouth covered https://doi.org/10.1111/ina.12109

[31] Observing and quantifying airflows in the infection control of aerosol- and airborne-transmitted diseases: an overview of approaches https://doi.org/10.1016/j.jhin.2010.09.037

[32] Han DW, Shim YH, Shin CS, Lee YW, Lee JS, Ahn SW. Estimation of the length of the naresvocal cord. Anesth Analg. (2005) 100:1533-5. 10.1213/01.ANE.0000149900.68354.33

[33] Varshney M, Sharma K, Kumar R, Varshney PG. Appropriate depth of placement of oral endotracheal tube and its possible determinants in Indian adult patients. Indian $\mathrm{J}$ Anaesth. (2011) 55:488-93. 10.4103/0019-5049.89880 

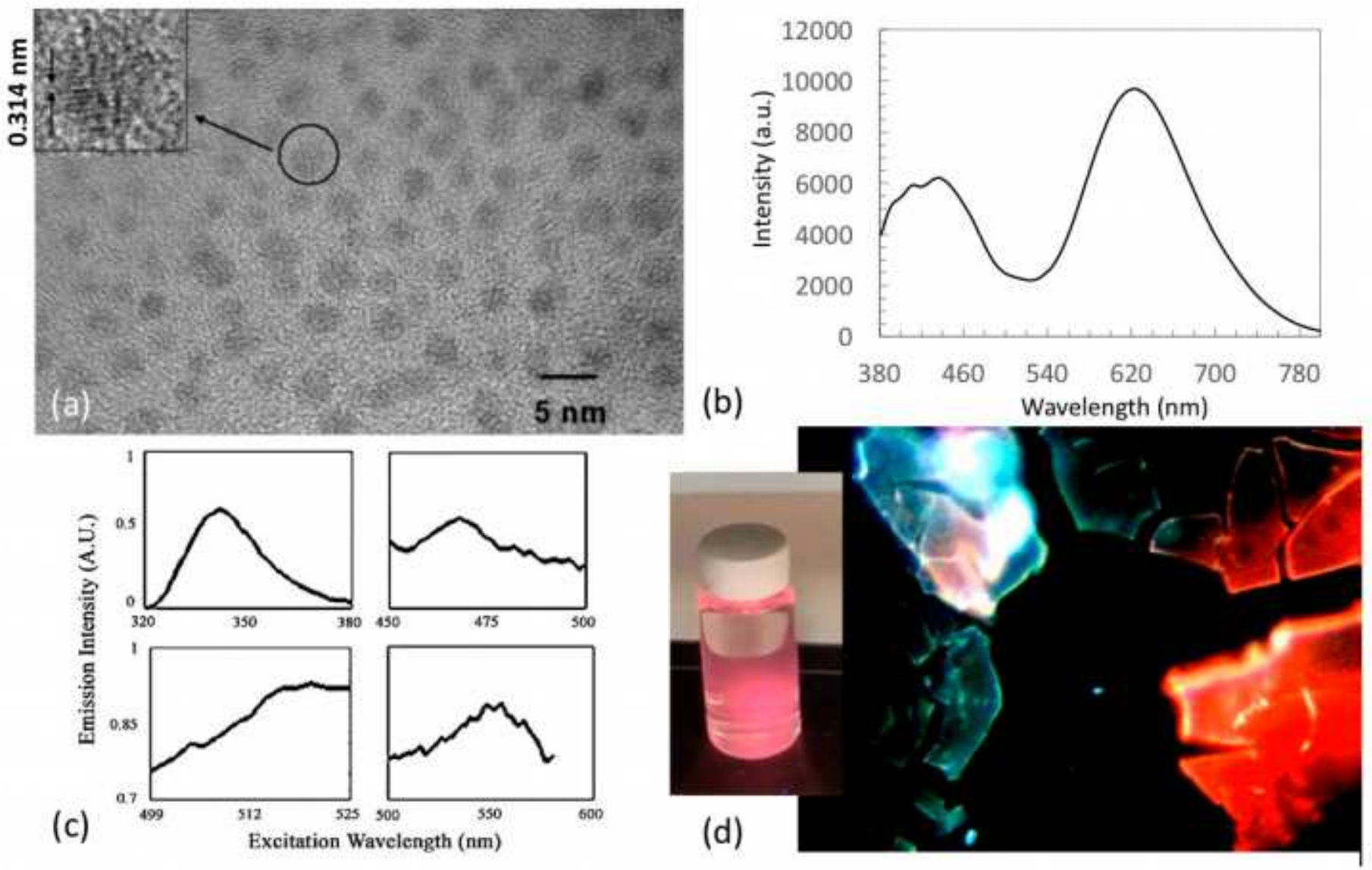

\section{Figure 1}

(a)Transmission electron microscopic image of individual Si NPs and (b) Emission spectrum of the Si NPs colloid showing a red-orange band and a smaller blue-green band under irradiation at $355 \mathrm{~nm}$ UV radiation. (c) emission spectra of 1, 1.67, 2.2, and $2.9 \mathrm{~nm}$ particles with excitation at their respective confinement bandgaps [23]. (d) The Si NPs solution can be seen to be dominated by brightly red-orange luminescence under $365 \mathrm{~nm}$ irradiation and the self-assembly on a surface of same size (emitting same band) nanoparticles 


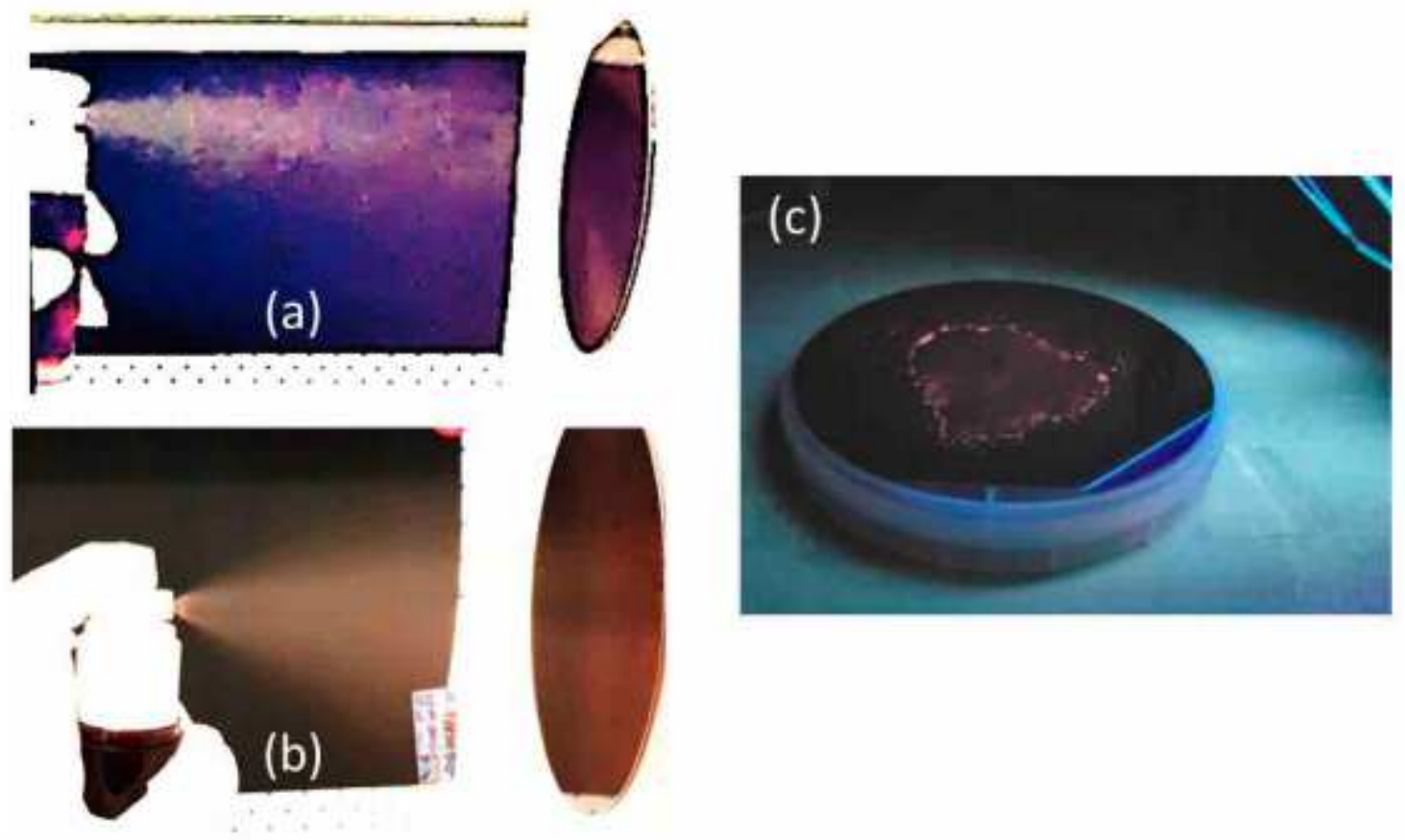

Figure 2

(a) and (b) Optimizing the spray coating pattern on a Si wafer. The spray cloud under exposure of UV radiation with a wavelength of $365 \mathrm{~nm}$ (c) An example of a Si wafer under UV irradiation after spraying the Si NPs. 


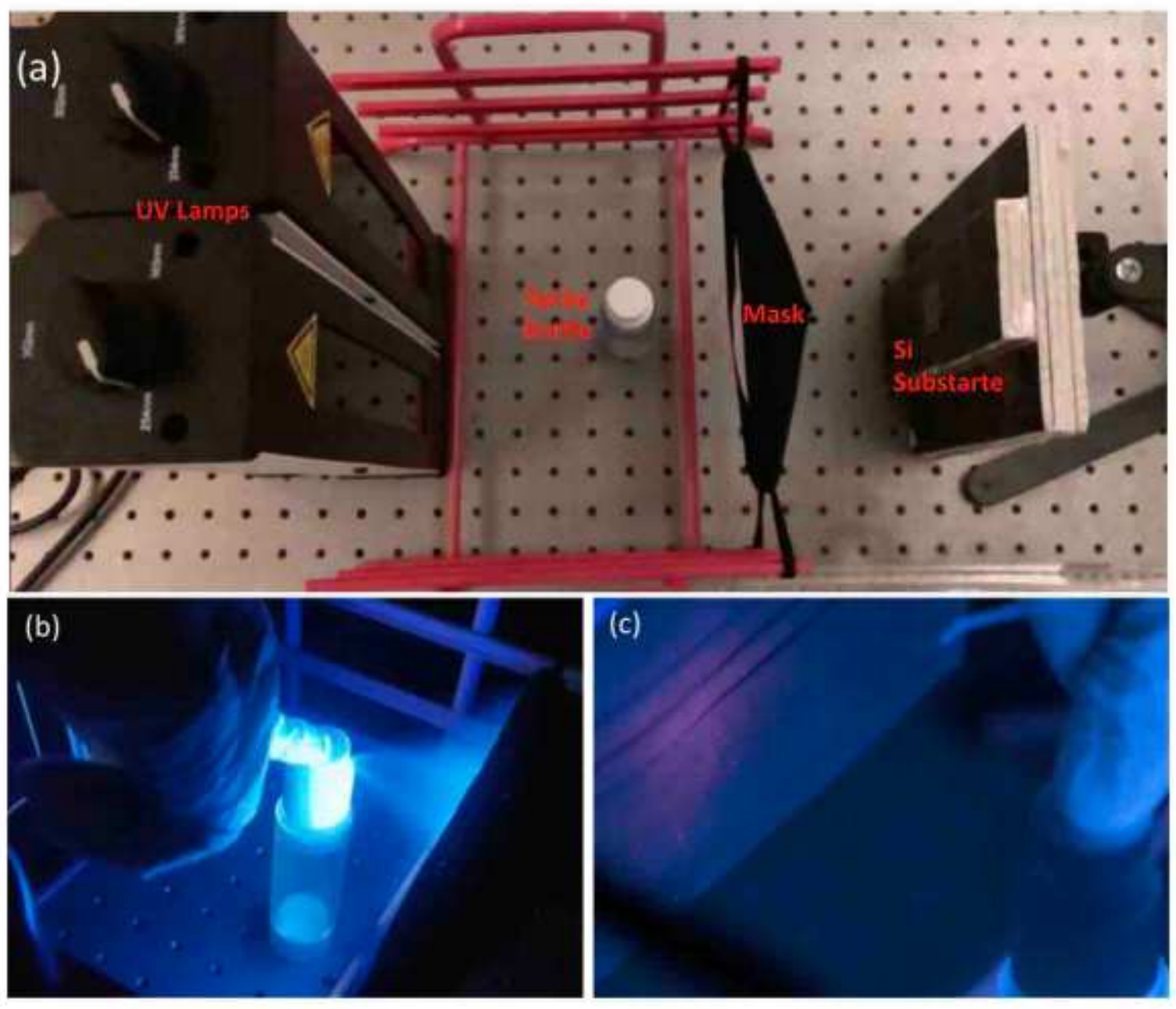

Figure 3

(a) Top view of the setup used to spray the masks (b) Spray pattern from the spray bottle (c) Spraying the mask under the UV lamp 

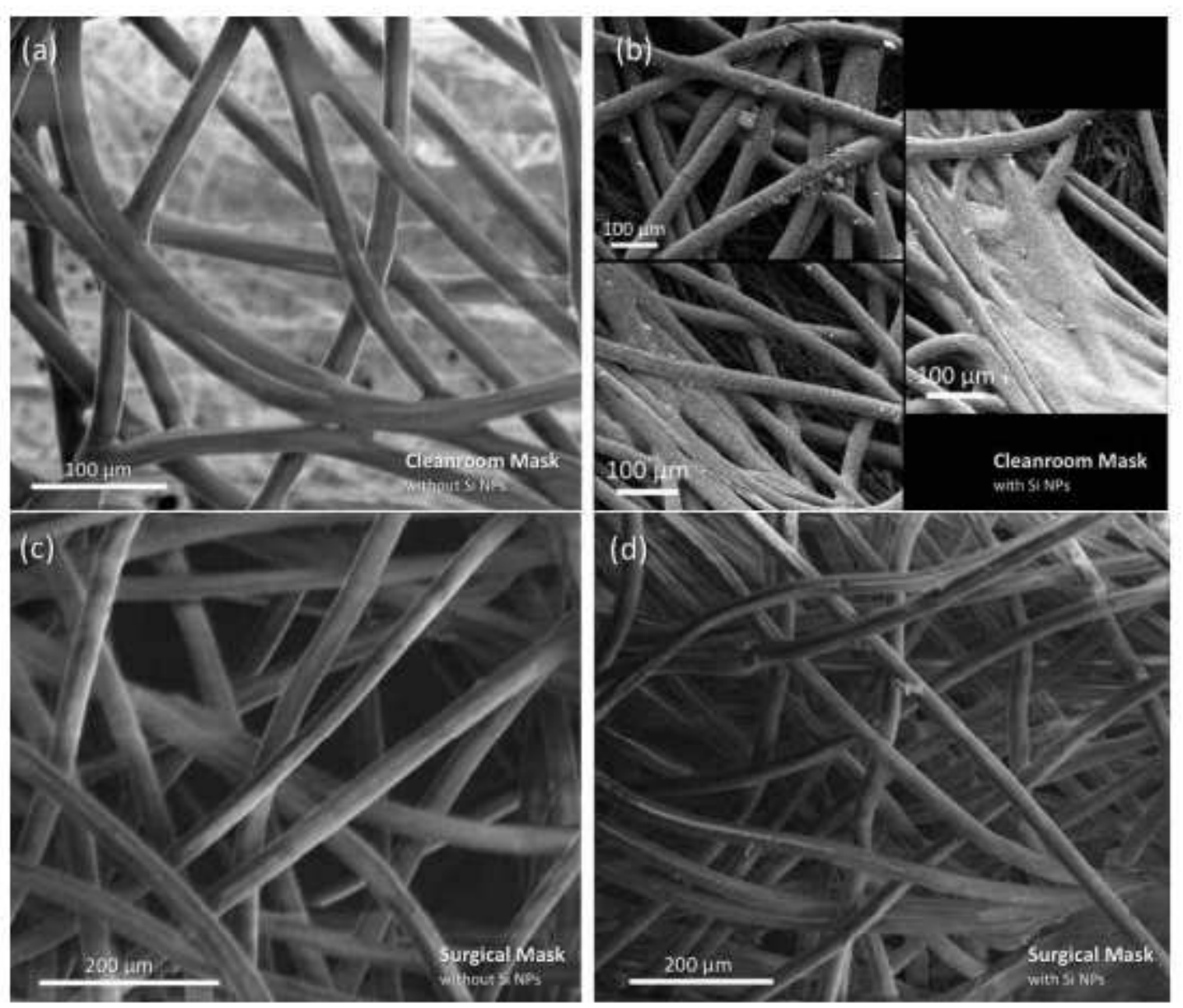

\section{Figure 4}

Scanning electron microscope image of the (a-b) cleanroom and (c-d) surgical mask with and without Si nano particles.
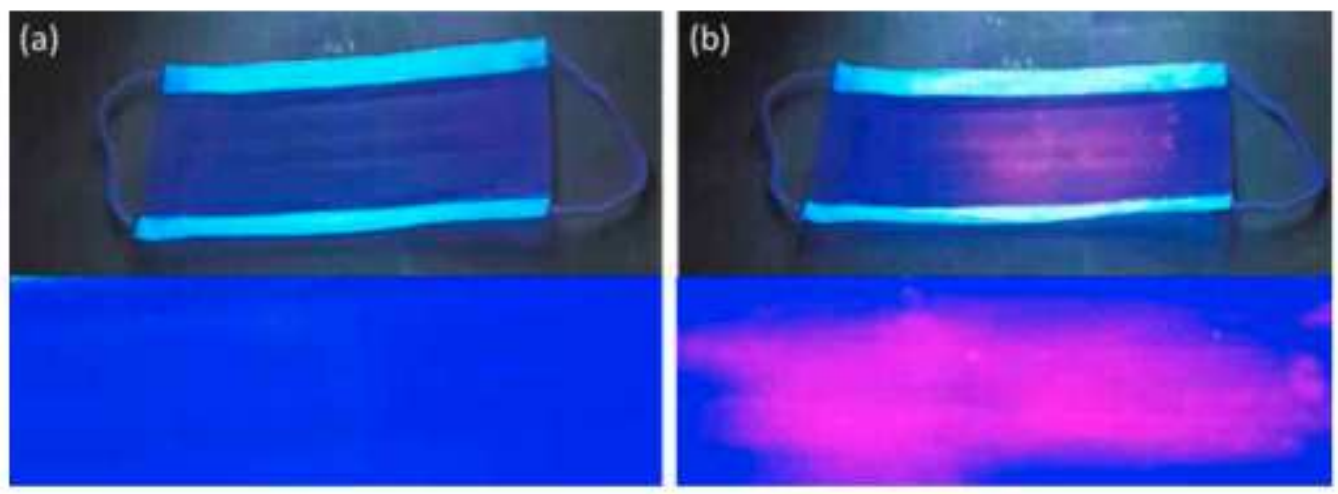

\section{Figure 5}

Surgical mask under UV (a) before spraying and (b) after spraying the Si NPs 


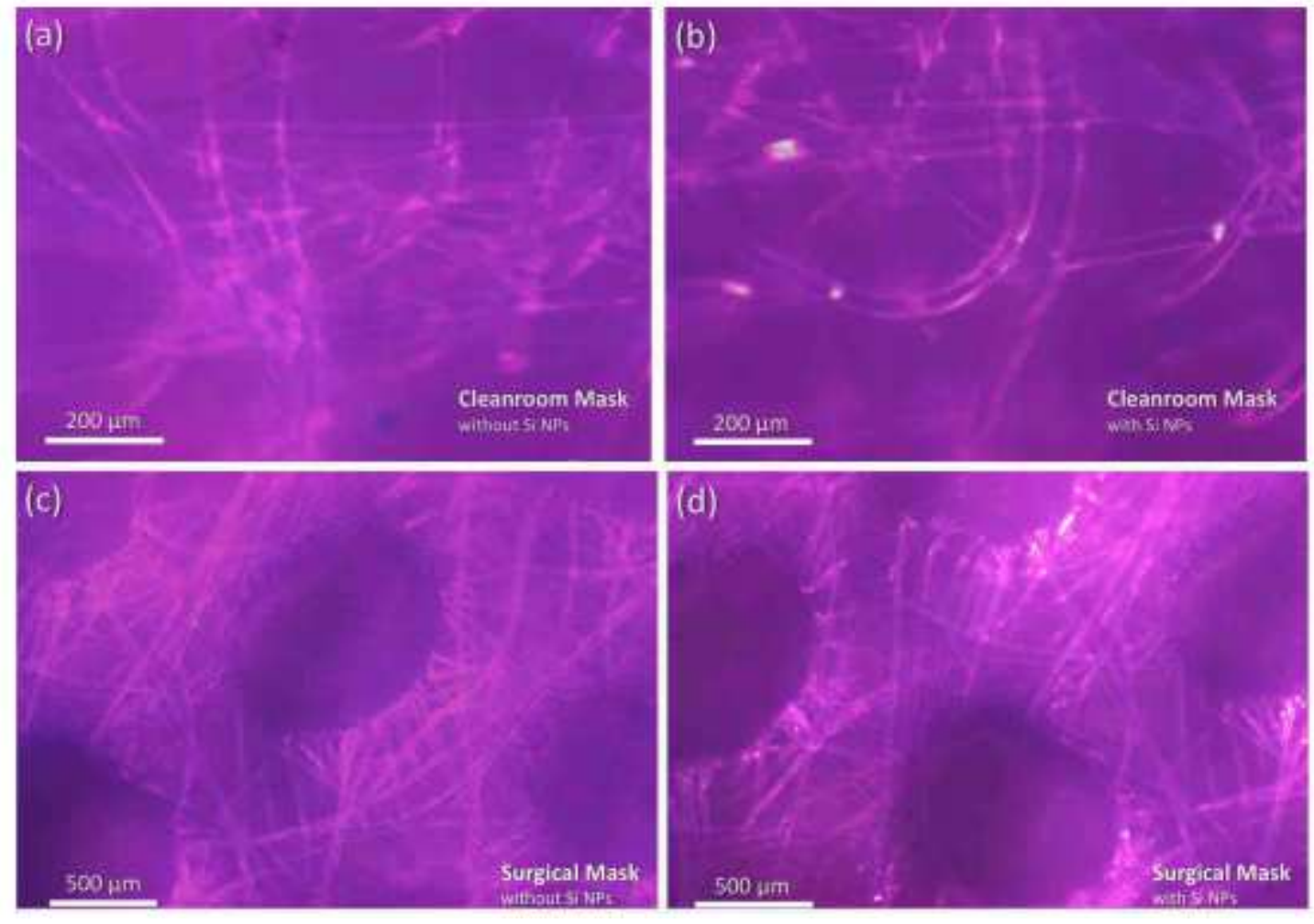

Figure 6

An optical image of the (a-b) surgical and (c-d) cleanroom mask under UV with and without Si nanoparticles. 


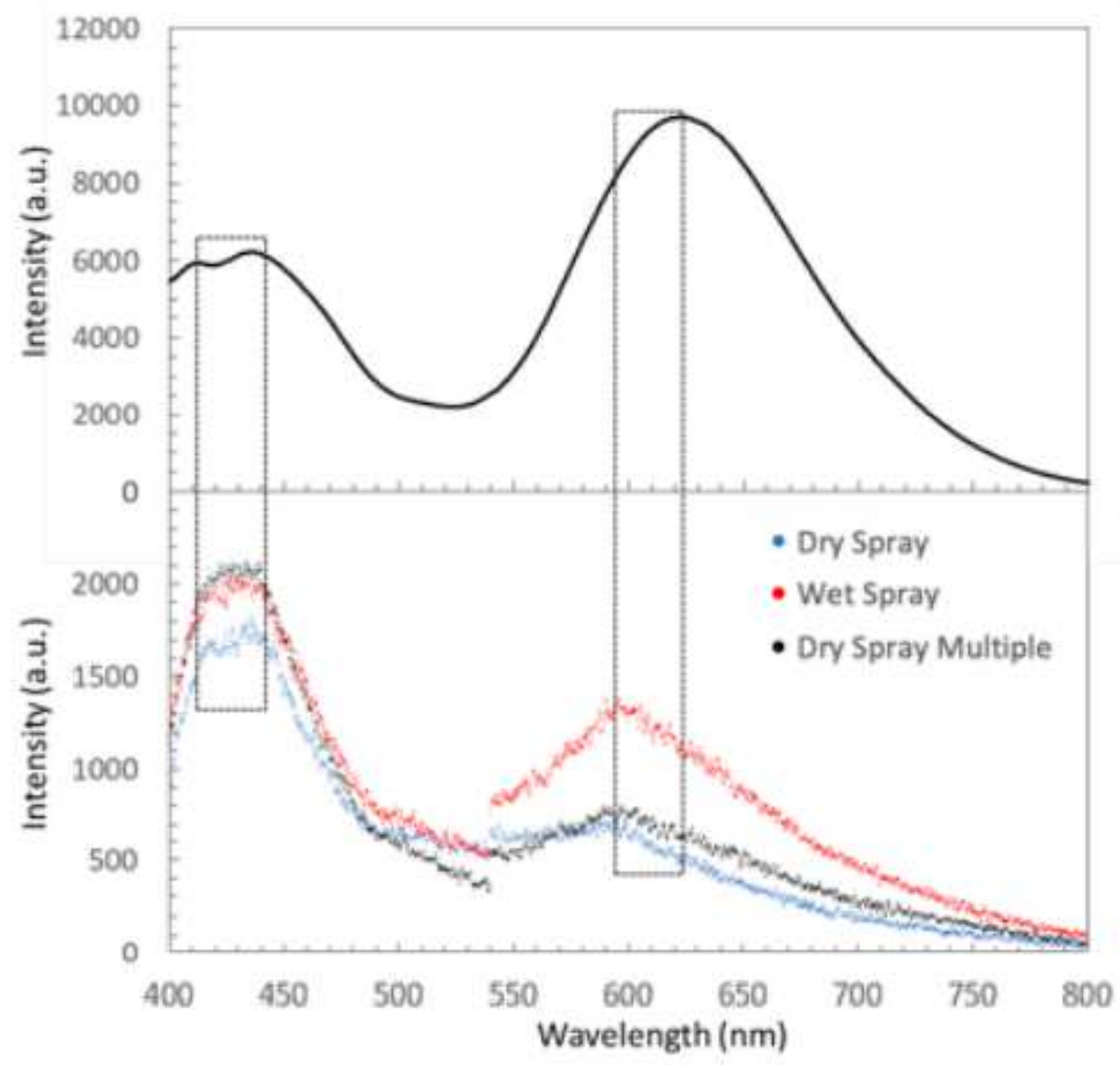

Figure 7

Luminescence as a function of wavelength on (top) Si-NPs solution and (bottom) mask with Si-NPs. The excitation wavelength is fixed at $355 \mathrm{~nm}$ while the emission arm is scanned over the range $370-760 \mathrm{~nm}$. The discontinuity in the spectra at is due to a filter change.

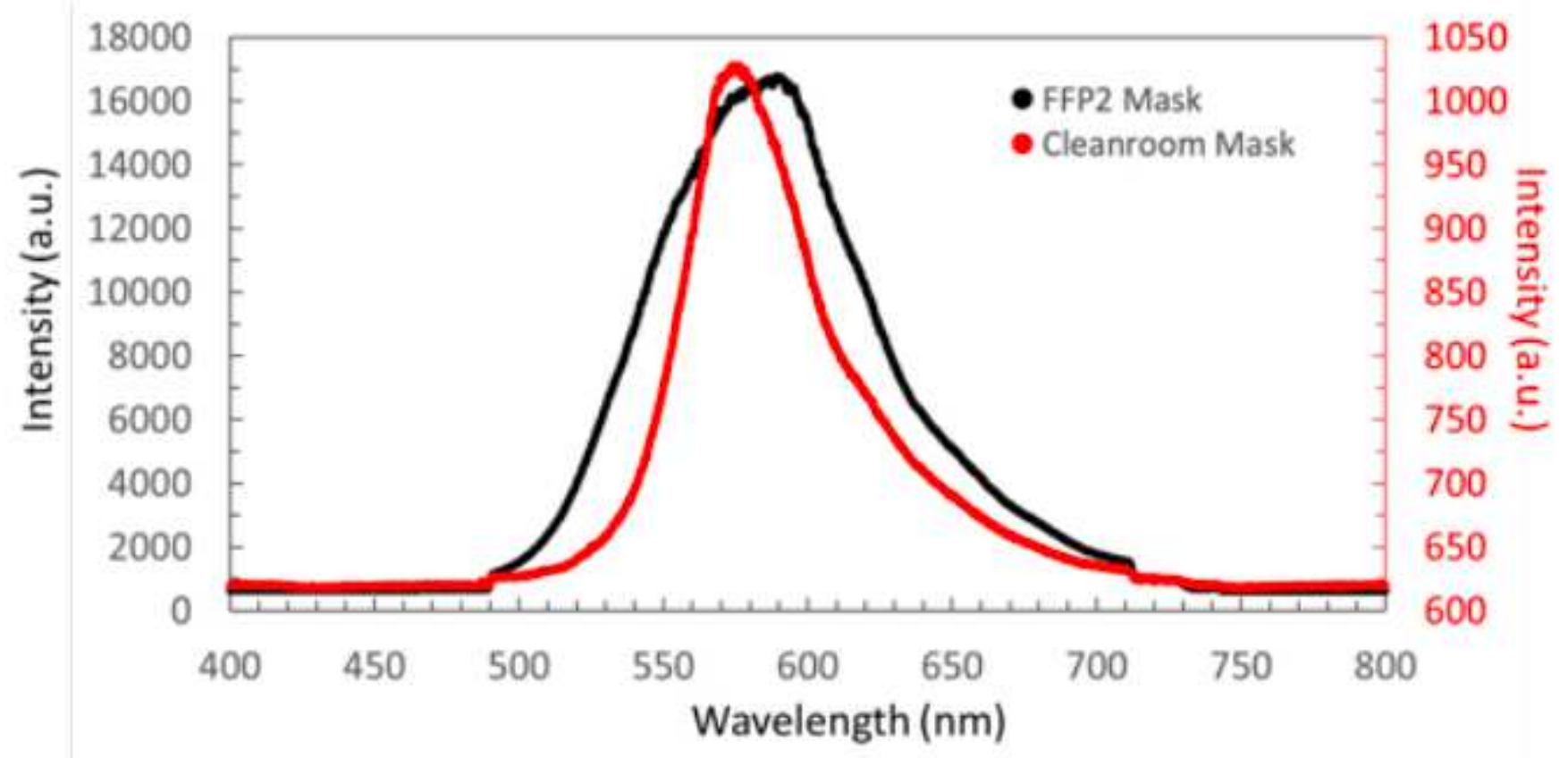


Figure 8

The PL spectra of coated FFP2 Mask and Cleanroom Mask at 488nm

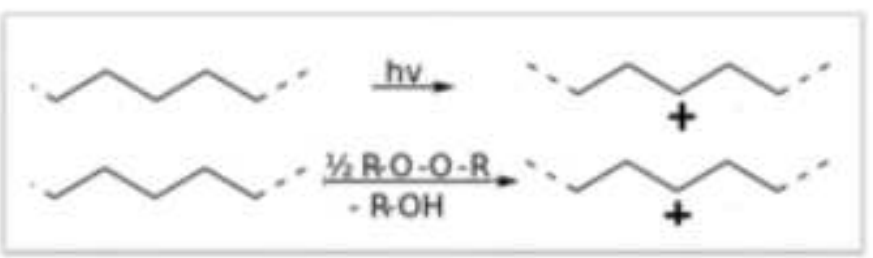<smiles>CCCC(CCC)C(CCC)CCC</smiles>

peroxide crosslinking /

irradiation crosslinking

\section{Figure 9}

Under UV irradiation or action of hydrogen peroxide, the softened of the hydrogen bond in the polyethylene fiber causing the two fibers to crosslink through $\mathrm{C}-\mathrm{C}$ bonding.
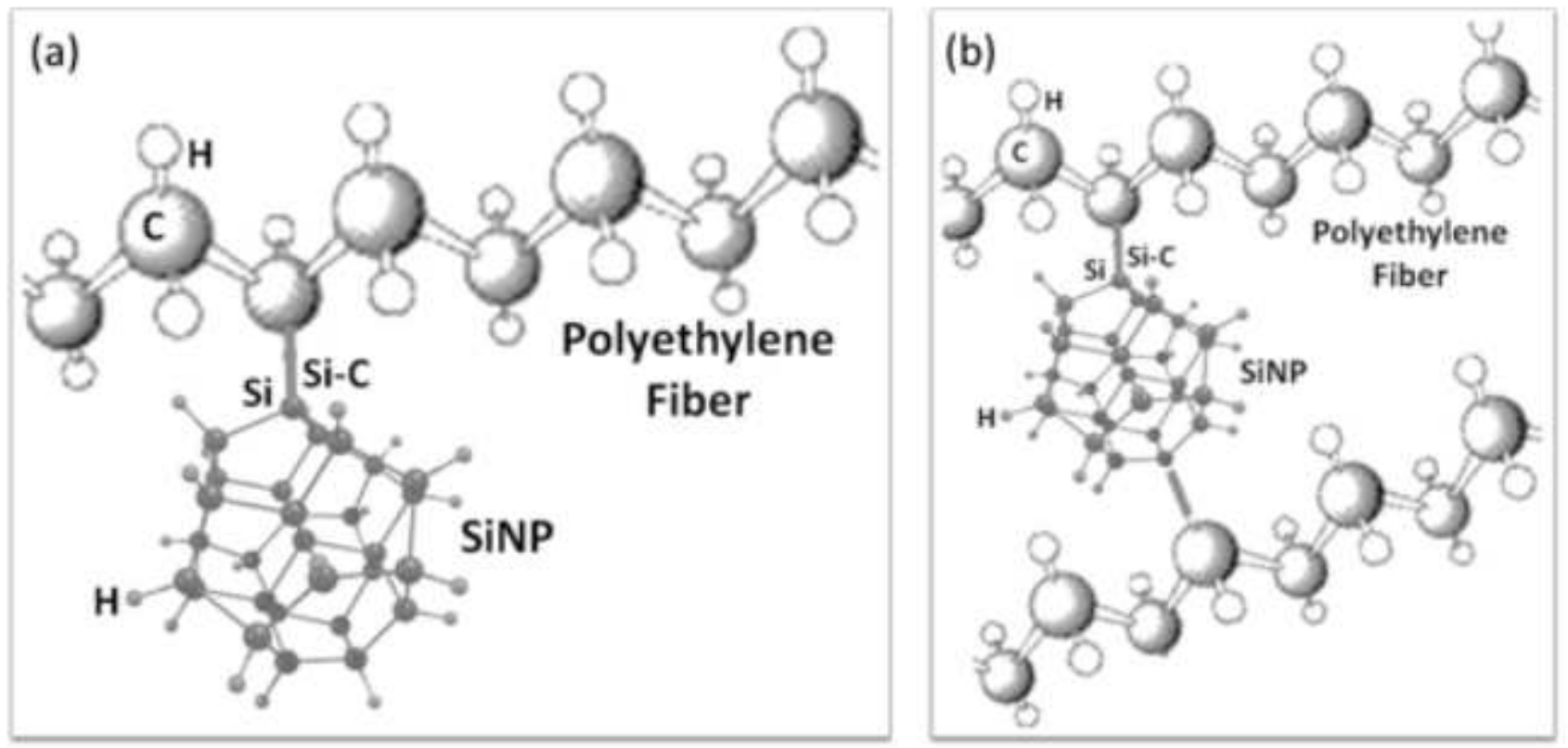

Figure 10

(a) The Si-C bond form between the bare silicon and the carbon atoms in PE. (b) The Si NP interacting with multiple PE chain while the nanoparticle acts a secondary cross linker. 


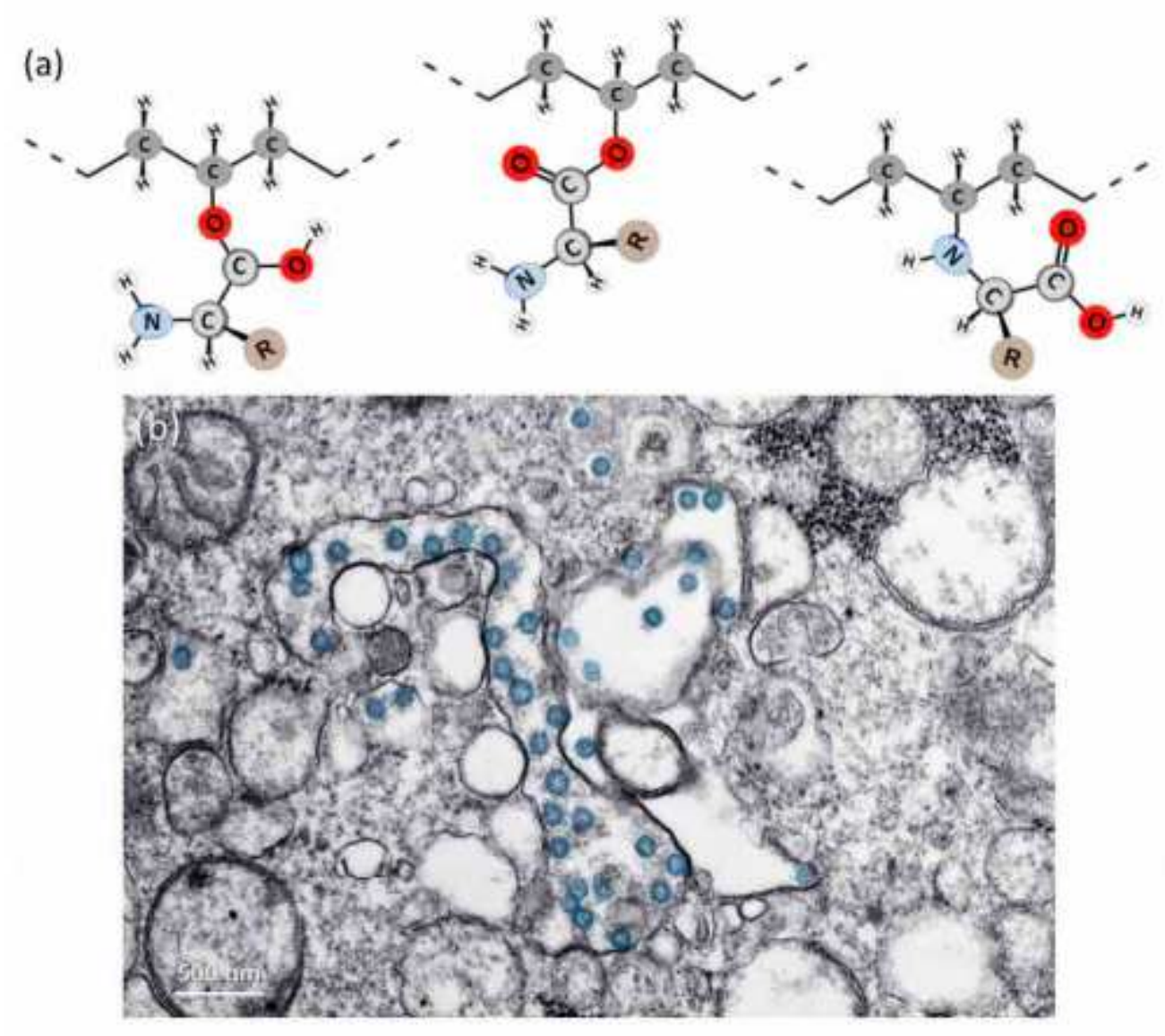

\section{Figure 11}

(a) The PE chemical attachments via the covalent bonds C-O and C-N (b) TEM image of COVID-19 virus. The spherical viral particles, colorized blue. 